\title{
FREE BOUNDARY PROBLEM FOR THE EQUATIONS OF MAGNETOHYDRODYNAMIC INCOMPRESSIBLE VISCOUS FLUID
}

Abstract. The existence of a global motion of magnetohydrodynamic fluid in a domain bounded by a free surface and under the external electrodynamic field is proved. The motion is such that the velocity and magnetic field are small in the $H^{3}$-space.

1. Introduction. In this paper we prove the existence of global solutions to the equations describing the motion of a magnetohydrodynamic incompressible viscous fluid in a domain $\Omega_{t} \subset \mathbb{R}^{3}$ bounded by a free surface $S_{t}$. In the domain $D_{t} \subset \mathbb{R}^{3}$ which is exterior to $\Omega_{t}$ we have a gas under constant pressure $p_{0}$. Moreover in $D_{t}$ we have an electromagnetic field which is generated by some currents which are located on a fixed boundary $B$ of $D_{t}$.

In the domain $\Omega_{t}$ the motion is described by the following problem:

$$
\begin{array}{ll}
v_{t}+v \cdot \nabla v-\operatorname{div} \mathbb{T}(v, p)-\mu_{1} \stackrel{1}{H} \cdot \nabla \stackrel{1}{H}+\mu_{1} \nabla \frac{H^{2}}{2}=f & \text { in } \widetilde{\Omega}^{T}, \\
\operatorname{div} v=0, & \text { in } \widetilde{\Omega}^{T}, \\
\stackrel{1}{H}=-\operatorname{rot} \stackrel{1}{E} & \text { in } \widetilde{\Omega}^{T}, \\
\mu_{1}, \stackrel{1}{H}=\sigma_{1}\left(\stackrel{1}{E}+\mu_{1} v \times \stackrel{1}{H}\right) & \text { in } \widetilde{\Omega}^{T}, \\
\operatorname{rot} \stackrel{1}{H}=0 & \text { in } \widetilde{\Omega}^{T}, \\
\operatorname{div}\left(\mu_{1}\right)=0
\end{array}
$$

where $\widetilde{\Omega}^{T}=\bigcup_{0 \leq t \leq T} \Omega_{t} \times\{t\}, v=v(x, t)$ is the velocity of fluid, $p=p(x, t)$ is the pressure, $\stackrel{1}{H}=\stackrel{1}{H}(x, t)$ is the magnetic field, $f=f(x, t)$ is the external

2000 Mathematics Subject Classification: 35A05, 35R35, 76N10.

Key words and phrases: free boundary, global existence, Sobolev spaces, magnetohydrodynamic incompressible fluid.

Supported by KBN grant no. 2PO3A00223. 
force field per unit mass, $\mu_{1}$ is the constant magnetic permeability, $\sigma_{1}$ is the constant electric conductivity, $\stackrel{1}{E}=\stackrel{1}{E}(x, t)$ is the electric field, and

$$
\mathbb{T}(v, p)=\left\{\nu\left(\partial_{x_{i}} v_{j}+\partial_{x_{j}} v_{i}\right)-p \delta_{i j}\right\}
$$

is the stress tensor, where $\nu$ is the viscosity of the fluid. Moreover,

$$
\mathbb{D}(v)=\left\{\nu\left(\partial_{x_{i}} v_{j}+\partial_{x_{j}} v_{i}\right)\right\}
$$

is the dilatation tensor.

In the domain $D_{t}$ in which there is a dielectric (gas) we assume that there is no fluid motion inside $(v=0)$. Therefore we have the electromagnetic field only, described by the following system:

$$
\begin{array}{ll}
\mu_{2} \stackrel{2}{H}_{t}=-\operatorname{rot} \stackrel{2}{E} & \text { in } \widetilde{D}^{T}, \\
\operatorname{rot} \stackrel{2}{H}=\sigma_{2} \stackrel{2}{H} & \text { in } \widetilde{D}^{T}, \\
\operatorname{div}\left(\mu_{2} \stackrel{2}{H}=0\right. & \text { in } \widetilde{D}^{T},
\end{array}
$$

where $\widetilde{D}^{T}=\bigcup_{0 \leq t \leq T} D_{t} \times\{t\}$.

On $S_{t}=\partial \Omega_{t} \cap \partial D_{t}$ we assume the following transmission and boundary conditions:

$$
\begin{array}{lr}
\mathbb{T}(v, p) n=\left(-p_{0} I-\mu_{1} \stackrel{1}{H} \otimes \stackrel{1}{H}+\mu_{1} \frac{\stackrel{1}{H}^{2}}{2} I\right) n & \text { on } \widetilde{S}^{T}, \\
\frac{1}{\sigma_{1}} \stackrel{1}{H}=\frac{1}{\sigma_{2}} \stackrel{2}{H} & \text { on } \widetilde{S}^{T}, \\
\stackrel{1}{E} \cdot \tau_{\alpha}=\stackrel{2}{E} \cdot \tau_{\alpha}, \quad \alpha=1,2, & \text { on } \widetilde{S}^{T}, \\
v \cdot n=-\frac{\phi_{t}}{|\nabla \phi|} & \text { on } \widetilde{S}^{T},
\end{array}
$$

where $\widetilde{S}^{T}=\bigcup_{0<t<T} S_{t} \times\{t\}, n$ is the unit outward vector to $\Omega_{t}$ and normal to $S_{t}, \tau_{\alpha}, \alpha=1,2$, is the tangent vector to $S_{t}$, and $\phi(x, t)=0$ describes $S_{t}$ at least locally.

Next we assume the boundary conditions on $B$ :

$$
\begin{array}{ll}
\stackrel{2}{H}=H_{*} & \text { on } B, \\
\stackrel{2}{E}=E_{*} & \text { on } B .
\end{array}
$$

Finally, we assume the initial conditions

$$
\begin{array}{rlrl}
\left.\Omega_{t}\right|_{t=0}=\Omega, & \left.S_{t}\right|_{t=0}=S, & \left.D_{t}\right|_{t=0}=D, \\
\left.v\right|_{t=0}=v_{0}, & \left.\stackrel{1}{H}\right|_{t=0}=\stackrel{1}{H}, & \text { in } \Omega, \\
\left.\stackrel{2}{H}\right|_{t=0}=\stackrel{2}{H}_{0}, & \text { in } D .
\end{array}
$$


Now we make some comments on the literature concerning free boundary problems for the nonstationary incompressible Navier-Stokes system. Local existence of solutions in the case without surface tension was proved in Hölder and Sobolev anisotropic spaces by V. A. Solonnikov in [4, 5]. To prove the existence of solutions of corresponding linear problems in Hölder and in Sobolev spaces the potential theory techniques were used (see [6, 7], respectively). In [4] V. A. Solonnikov showed the existence of global motions of a viscous incompressible fluid bounded by a free surface. The proof was based on the Korn inequality. To prove the existence of solutions in the case of surface tension V. A. Solonnikov used the anisotropic Sobolev-Slobodetski1 spaces $W_{2}^{l, l / 2}$ with noninteger positive $l$. In all papers by Solonnikov, Lagrangian coordinates are used.

To prove existence of solutions to the above problem we introduce the Lagrangian coordinates $\xi \in \Omega$. They are the initial data for the Cauchy problem

$$
\frac{d x}{d t}=v(x, t),\left.\quad x\right|_{t=0}=\xi \in \Omega .
$$

Therefore $x_{v}(\xi, t)=\xi+\int_{0}^{t} \bar{v}(\xi, \tau) d \tau$, where

$$
\bar{v}(\xi, t)=v\left(x_{v}(\xi, t), t\right) .
$$

To introduce the Lagrangian coordinates in $D_{t}$ we extend $v$ onto $D_{t}$. Let us denote the extended function by $v^{\prime}$. Then we define $\xi \in D$ to be the Cauchy data to the problem

$$
\frac{d x}{d t}=v^{\prime}(x, t),\left.\quad x\right|_{t=0}=\xi \in D .
$$

Therefore $x_{v^{\prime}}(\xi, t)=\xi+\int_{0}^{t} \bar{v}^{\prime}(\xi, \tau) d \tau$, where $\bar{v}^{\prime}(\xi, t)=v^{\prime}\left(x_{v^{\prime}}(\xi, t), t\right)$. Then by $(1.1)_{5}$,

$$
\begin{aligned}
& \Omega_{t}=\left\{x \in \mathbb{R}^{3}: x=x_{v}(\xi, t), \xi \in \Omega\right\}, \\
& S_{t}=\left\{x \in \mathbb{R}^{3}: x=x_{v}(\xi, t), \xi \in S\right\} .
\end{aligned}
$$

Since $S_{t}$ is determined at least locally by the equation $\phi(x, t)=0, S$ is described by $\left.\phi\left(x_{v}(\xi, t), t\right)\right|_{t=0}=0$. Moreover, we have

$$
\bar{n}_{v}=n\left(x_{v}(\xi, t), t\right)=\left.\frac{\nabla_{x} \phi(x, t)}{\left|\nabla_{x} \phi(x, t)\right|}\right|_{x=x_{v}(\xi, t)} .
$$

To simplify considerations we introduce the following notation:

$$
\begin{aligned}
& \|u\|_{l, Q}=\|u\|_{H^{l}(Q)}, \quad Q \in\{\Omega, S, D, \Pi, B\}, \quad 0 \leq l \in \mathbb{Z}, \\
& \|u\|_{k, p, q, Q^{T}}=\|u\|_{L_{q}\left(0, T, W_{p}^{k}(Q)\right)}, \quad Q \in\{\Omega, S, D, \Pi, B\}, \\
& \quad p, q \in[1, \infty], \quad 0 \leq k \in \mathbb{Z},
\end{aligned}
$$


where $Q^{t}=Q \times(0, t)$,

$$
|u|_{p, Q}=\|u\|_{L_{p}(Q)}, \quad Q \in\{\Omega, S, D, \Pi, B\}, \quad p \in[1, \infty] .
$$

2. Weak solutions. Weak solutions to problem (1.1)-(1.7) are formulated in Lagrangian coordinates.

DeFinition 2.1. A weak solution of problem (1.1)-(1.7) is a pair of functions $\bar{v}, \bar{H}$ which satisfy the integral identities

$$
\begin{aligned}
\int_{0}^{T} \int_{\Omega}\left(-\bar{v} \cdot \bar{\varphi}_{t}+\mathbb{D}_{v}(\bar{v}) \cdot \mathbb{D}_{v}(\bar{\varphi})\right) d \xi d t & \\
& \quad-\int_{0}^{T} \int_{\Omega}\left(\mu_{1} \frac{1}{H} \cdot \nabla_{v} \frac{1}{H} \cdot \bar{\varphi}-\mu_{1} \nabla_{v} \frac{\bar{H}^{2}}{2} \cdot \bar{\varphi}\right) d \xi d t
\end{aligned}
$$

$=\int_{0}^{T} \int_{\Omega} \bar{f} \cdot \bar{\varphi} d \xi d t+\int_{0}^{T} \int_{S}\left(-p_{0} I-\mu_{1} \frac{1}{H} \otimes \frac{1}{\bar{H}}+\mu_{1} \frac{\bar{H}^{2}}{2} I\right) \bar{n} \cdot \bar{\varphi} d \xi_{S} d t-\int_{\Omega} \bar{v}_{0} \cdot \bar{\varphi}(0) d \xi$,

$$
\begin{array}{r}
\int_{0}^{T} \int_{\Pi}\left(-\mu \bar{H} \cdot \bar{\psi}_{t}-\mu \bar{v} \cdot \nabla_{v} \bar{H} \cdot \bar{\psi}+\frac{1}{\sigma} \operatorname{rot}_{v} \bar{H} \cdot \operatorname{rot}_{v} \bar{\psi}\right) d \xi d t \\
-\int_{0}^{T} \int_{\Omega} \mu_{1}(\bar{v} \times \bar{H}) \cdot \operatorname{rot}_{v} \bar{\psi} d \xi d t \\
=\frac{1}{\sigma_{2}} \int_{0}^{T} \int_{B}\left(\bar{n}_{v} \times \bar{E}_{*}\right) \cdot \bar{\psi} d \xi_{B} d t-\mu \int_{\Pi} \bar{H}_{0} \cdot \bar{\psi}(0) d \xi
\end{array}
$$

where $\varphi, \psi$ are sufficiently regular and $\varphi(x, T)=\psi(x, T)=0, \bar{n}_{v}$ is the unit outward vector normal to $S$ or $B$.

In (2.1), (2.2) we use the notation $\bar{A}(\xi, t)=A\left(x_{v}(\xi, t), t\right), \bar{H}_{\mid \Omega}=\frac{1}{\bar{H}}$, $\bar{H}_{\mid D}=\stackrel{2}{\bar{H}}, \sigma_{\mid \Omega}=\sigma_{1}, \sigma_{\mid D}=\sigma_{2}, \Pi=\Omega \cup D, \mu_{\mid \Omega}=\mu_{1}, \mu_{\mid D}=\mu_{2}, v$ in (2.2) is an extension onto $\Pi$,

$$
\begin{aligned}
& \mathbb{D}_{v}(\bar{v})=\left\{\nu\left(\partial_{x_{i}} \xi_{k} \nabla_{\xi_{k}} \bar{v}_{j}+\partial_{x_{j}} \xi_{k} \nabla_{\xi_{k}} \bar{v}_{i}\right)\right\}, \quad \operatorname{rot}_{v} \bar{v}=\nabla_{v} \times \bar{v} \\
& \nabla_{v}=\partial_{x} \xi_{i} \nabla_{\xi_{i}}, \quad \operatorname{div}_{v} \bar{v}=\nabla_{v} \cdot \bar{v}=\partial_{x_{i}} \xi_{k} \nabla_{\xi_{k}} \bar{v}_{i}, \quad \partial_{\xi_{i}}=\nabla_{\xi_{i}} .
\end{aligned}
$$

Let $A$ be the Jacobi matrix of the transformation $x=x_{v}(\xi, t)$. Then $\operatorname{det} A=$ $\exp \left(\int_{0}^{t} \operatorname{div}_{v} \bar{v} d \tau\right)=1$.

Moreover

$$
x_{\xi_{j}}^{i}=\delta_{i j}+\int_{0}^{t} \partial_{\xi_{j}} \bar{v}_{i}(\xi, \tau) d \tau \quad \text { and } \quad \xi_{x}=x_{\xi}^{-1} .
$$


Hence we get

$$
\begin{aligned}
\sup _{\xi \in \Omega}\left|x_{\xi}\right| & \leq 1+\sup _{\xi \in \Omega} \int_{0}^{t}\left|\bar{v}_{\xi}(\xi, \tau)\right| d \tau \leq 1+c \int_{0}^{t}\|\bar{v}\|_{3, \Omega} d \tau \\
& \leq 1+c \sqrt{t} \sqrt{\int_{0}^{t}\|\bar{v}\|_{3, \Omega}^{2} d \tau} \leq 1+c \sqrt{t}\|\bar{v}\|_{3,2,2, \Omega^{t}} .
\end{aligned}
$$

Therefore $\sup _{x \in \Omega_{t}}\left|\xi_{x}\right| \leq \alpha(a)$, where $a=\sqrt{t}\|\bar{v}\|_{3,2,2, \Omega^{t}}$ and $\alpha$ is an increasing positive function.

To prove the existence of a solution to the above problem we linearize $(2.1),(2.2)$ to the form

$$
\begin{aligned}
& \int_{0}^{T} \int_{\Omega}\left(-\bar{v} \bar{\varphi}_{t}+\mathbb{D}_{u}(\bar{v}) \cdot \mathbb{D}_{u}(\bar{\varphi})\right) d \xi d t \\
& -\int_{0}^{T} \int_{\Omega}\left(\mu_{1} \bar{H}^{\prime} \cdot \nabla_{u} \bar{H}^{\prime} \cdot \bar{\varphi}-\mu_{1} \nabla_{u} \frac{\bar{H}^{\prime 2}}{2} \cdot \bar{\varphi}\right) d \xi d t=\int_{0}^{T} \int_{\Omega} \bar{f} \cdot \bar{\varphi} d \xi d t \\
& +\int_{0}^{T} \int_{S}\left(-p_{0} I-\mu_{1} \frac{1}{H} \otimes \frac{1}{H}+\mu_{1} \frac{\bar{H}^{2}}{2} I\right) \bar{n}_{u} \cdot \bar{\varphi} d \xi_{S} d t-\int_{\Omega} \bar{v}_{0} \cdot \bar{\varphi}(0) d \xi, \\
& \int_{0}^{T} \int_{\Pi}\left(-\mu \bar{H} \cdot \bar{\psi}_{t}-\mu \bar{u} \cdot \nabla_{u} \bar{H} \cdot \bar{\psi}+\frac{1}{\sigma} \operatorname{rot}_{u} \bar{H} \cdot \operatorname{rot}_{u} \bar{\psi}\right) d \xi d t \\
& -\int_{0}^{T} \int_{\Omega} \mu_{1}\left(\bar{u} \times \frac{1}{H}\right) \cdot \operatorname{rot}_{u} \bar{\psi} d \xi d t \\
& =\frac{1}{\sigma_{2}} \int_{0}^{T} \int_{B}\left(\bar{n}_{u} \times \bar{E}_{*}\right) \cdot \bar{\psi} d \xi_{B} d t-\mu \int_{\Pi} \bar{H}_{0} \cdot \bar{\psi}(0) d \xi
\end{aligned}
$$

where $\frac{1}{H^{\prime}}$ and $u$ with $\operatorname{div} u=0$ are given functions.

Similarly to [1], [2] we prove

THEOREM 2.1. Assume that $\bar{v}_{0} \in H^{2}(\Omega) ; \bar{v}_{t}(0), \bar{v}_{t t}(0) \in L_{2}(\Omega) ; \bar{f}_{t}, \bar{f}_{t t} \in$ $L_{2}\left(0, T, L_{2}(\Omega)\right) ; \bar{f} \in L_{2}\left(0, T, H^{2}(\Omega)\right) ; \bar{H}_{0} \in H^{2}(\Pi) ; \bar{H}_{t}(0) \in H^{1}(\Pi) ; \bar{E}_{*} \in$ $L_{\infty}\left(0, T, H^{1}(B)\right) ; \bar{E}_{* t}, \bar{H}_{* t t} \in L_{2}\left(0, T, L_{2}(B)\right) ; \bar{H}_{* t} \in L_{2}\left(0, T, H^{2}(B)\right) ; \bar{H}_{*} \in$ $L_{2}\left(0, T, H^{3}(B)\right), S, B \in H^{5 / 2}$. Then there exists $T^{*}>0$ such that for $T \leq T^{*}$ there exists a solution to problem (1.1)-(1.7) such that

$$
\begin{aligned}
& \bar{v} \in L_{2}\left(0, T, H^{3}(\Omega)\right) \cap L_{\infty}\left(0, T, H^{1}(\Omega)\right) ; \\
& \bar{v}_{t} \in L_{\infty}\left(0, T, H^{1}(\Omega)\right) \cap L_{2}\left(0, T, H^{2}(\Omega)\right) ; \\
& \bar{v}_{t t} \in L_{\infty}\left(0, T, L_{2}(\Omega)\right) \cap L_{2}\left(0, T, H^{1}(\Omega)\right) ;
\end{aligned}
$$




$$
\begin{aligned}
& \bar{p} \in L_{2}\left(0, T, H^{2}(\Omega)\right) ; \bar{p}_{t} \in L_{2}\left(0, T, H^{1}(\Omega)\right) ; \\
& \bar{H} \in L_{2}\left(0, T, H^{3}(\Pi)\right) \cap L_{\infty}\left(0, T, H^{1}(\Pi)\right) ; \\
& \bar{H}_{t} \in L_{\infty}\left(0, T, H^{1}(\Pi)\right) \cap L_{2}\left(0, T, H^{2}(\Pi)\right) ; \\
& \bar{H}_{t t} \in L_{\infty}\left(0, T, L_{2}(\Pi)\right) \cap L_{2}\left(0, T, H^{1}(\Pi)\right),
\end{aligned}
$$

where $\left(T^{*}\right)^{\gamma}(\varphi(0)+\beta) \leq b, b>0$ is a sufficiently small constant, $\gamma>0$ is a constant and

$$
\begin{aligned}
\beta= & \left\|\bar{E}_{*}\right\|_{0,2,2, B^{t}}^{2}+\left\|\bar{E}_{* t}\right\|_{0,2,2, B^{t}}^{2}+\left\|\bar{H}_{*}\right\|_{3,2,2, B^{t}}^{2}+\left\|\bar{H}_{* t}\right\|_{2,2,2, B^{t}}^{2} \\
& +\left\|\bar{H}_{* t t}\right\|_{0,2,2, B^{t}}^{2}+\left\|\bar{f}_{t}\right\|_{0,2,2, B^{t}}^{2}+\|\bar{f}\|_{1,2,2, B^{t}}^{2}, \\
\varphi(0)= & \sum_{i+k \leq 2}\left(\left\|\partial_{t}^{i} \bar{v}(0)\right\|_{k, \Omega}^{2}+\left\|\partial_{t}^{i} \bar{H}(0)\right\|_{k, \Pi}^{2}\right) .
\end{aligned}
$$

Moreover, if $\varphi(0), \beta$ are sufficiently small then

$$
\begin{aligned}
& \left\|\bar{v}_{t}\right\|_{1,2, \infty, \Omega^{T}}^{2}+\|\bar{v}\|_{2,2, \infty, \Omega^{T}}^{2}+\|\bar{v}\|_{3,2,2, \Omega^{T}}^{2}+\left\|\bar{v}_{t}\right\|_{2,2,2, \Omega^{T}}^{2} \\
& \quad+\left\|\bar{v}_{t t}\right\|_{1,2,2, \Omega^{T}}^{2}+\left\|\bar{p}^{\prime}\right\|_{2,2,2, \Omega^{T}}^{2}+\left\|\bar{p}_{t}\right\|_{1,2,2, \Omega^{T}}^{2}+\left\|\bar{H}_{t}\right\|_{1,2, \infty, \Pi^{T}}^{2} \\
& \quad+\|\bar{H}\|_{1,2, \infty, \Pi^{T}}^{2}+\|\bar{H}\|_{3,2,2, \Pi^{T}}^{2}+\left\|\bar{H}_{t}\right\|_{2,2,2, \Pi^{T}}^{2}+\left\|\bar{H}_{t t}\right\|_{1,2,2, \Pi^{T}}^{2} \\
& \leq c(\varphi(0)+\beta),
\end{aligned}
$$

where $\bar{p}^{\prime}=\bar{p}-p_{0}$.

First, in Section 3, we derive a differential inequality (3.23) which enables a step by step extension of the local solution of (1.1)-(1.7) from $[0, T]$ to $[0, \infty)$. In Section 4 we establish Korn type inequalities which are necessary to prove inequality (3.23). In Section 5 we prove the following

Main Theorem. Assume that $f=\int_{\Omega} v_{0} d x=\int_{\Omega} v_{0} \cdot \varphi_{i} d x=0, i=$ $1,2,3$, where $\varphi_{i}, i=1,2,3$, are defined in Lemma $4.1, H_{*} \in H^{3}(B), H_{* *} \in$ $H^{2}(B), H_{* t t} \in H^{1}(B), S_{t}, B \in H^{5 / 2},\left(v(0), p^{\prime}(0), H(0)\right) \in \mathcal{N}(0), \varphi(0) \leq \varepsilon_{1}$ where $\varepsilon_{1}$ is sufficiently small. Assume also that $\alpha(t) \leq e^{-\mu t}$, where $\mu>1 / 2$ is sufficiently large and $\alpha(t)$ is defined in Lemma 5.2. Then there exists a global solution of (1.1)-(1.7) such that $\left(v(t), p^{\prime}(t), H(t)\right) \in \mathcal{M}(t), t \in \mathbb{R}_{+}$, where $\mathcal{N}(0)$ and $\mathcal{M}(t)$ are defined in Section 5.

In Lemmas 3.1-3.12 we need

Lemma 2.1. For a solution of problem (1.1)-(1.7) we have

$$
\begin{aligned}
\left\|H_{t}\right\|_{1, \Omega}^{2} & \leq \alpha(a)\left(\left\|H_{t}\right\|_{1, \Omega_{t}}^{2}+\|H\|_{2, \Omega_{t}}^{2}\|v\|_{2, \Omega_{t}}^{2}\right), \\
\|\bar{H}\|_{i, \Omega}^{2} & \leq \alpha(a)\|H\|_{i, \Omega_{t}}^{2}, \quad i=1,2,3, \\
\|\bar{v}\|_{i, \Omega}^{2} & \leq \alpha(a)\|v\|_{i, \Omega_{t}}^{2}, \quad i=1,2,3, \\
\left\|\bar{H}_{t}\right\|_{2, \Omega}^{2} & \leq \alpha(a)\left[\left\|H_{t}\right\|_{2, \Omega_{t}}^{2}+\|H\|_{3, \Omega_{t}}^{2}\|v\|_{2, \Omega_{t}}^{2}+\|H\|_{2, \Omega_{t}}^{2}\|v\|_{3, \Omega_{t}}^{2}\right], \\
\left\|\bar{v}_{t}\right\|_{2, \Omega_{t}}^{2} & \leq \alpha(a)\left[\left\|v_{t}\right\|_{2, \Omega_{t}}^{2}+\|v\|_{3, \Omega_{t}}^{2}\|v\|_{2, \Omega_{t}}^{2}\right],
\end{aligned}
$$




$$
\begin{aligned}
\left\|\bar{H}_{t t}\right\|_{0, \Omega}^{2} \leq & \alpha(a)\left[\left\|H_{t t}\right\|_{0, \Omega_{t}}^{2}+\|v\|_{2, \Omega_{t}}^{2}\left(\left\|H_{t}\right\|_{1, \Omega_{t}}^{2}+\|H\|_{2, \Omega_{t}}^{2}\right)\right. \\
& \left.+\left\|v_{t}\right\|_{1, \Omega_{t}}^{2}\|H\|_{2, \Omega_{t}}^{2}+\|H\|_{2, \Omega_{t}}^{2}\|v\|_{2, \Omega_{t}}^{4}\right] \\
\left\|\bar{v}_{t t}\right\|_{0, \Omega}^{2} \leq & \alpha(a)\left[\left\|v_{t t}\right\|_{0, \Omega_{t}}^{2}+\|v\|_{2, \Omega_{t}}^{2}\left(\left\|v_{t}\right\|_{1, \Omega_{t}}^{2}+\|v\|_{2, \Omega_{t}}^{2}\right)+\|v\|_{2, \Omega_{t}}^{6}\right], \\
\left\|\bar{H}_{t t}\right\|_{1, \Omega}^{2} \leq & \alpha(a)\left[\left\|H_{t t}\right\|_{1, \Omega_{t}}^{2}+\|v\|_{2, \Omega_{t}}^{2}\left(\left\|H_{t}\right\|_{2, \Omega_{t}}^{2}+\|H\|_{3, \Omega_{t}}^{2}\right)\right. \\
& \left.+\|H\|_{3, \Omega_{t}}^{2}\left\|v_{t}\right\|_{2, \Omega_{t}}^{2}+\|H\|_{2, \Omega_{t}}^{2}\|v\|_{2, \Omega_{t}}^{4}\right] \\
\left\|\bar{v}_{t t}\right\|_{1, \Omega}^{2} \leq & \alpha(a)\left[\left\|v_{t t}\right\|_{1, \Omega_{t}}^{2}+\|v\|_{2, \Omega_{t}}^{2}\left(\left\|v_{t}\right\|_{2, \Omega_{t}}^{2}+\|v\|_{3, \Omega_{t}}^{2}\right)+\|v\|_{2, \Omega_{t}}^{6}\right],
\end{aligned}
$$

where $a=\sqrt{t}\|\bar{v}\|_{3,2,2, \Omega^{t}}$ and $\alpha$ is an increasing positive function.

Proof. Differentiating $\bar{H}(\xi, t)=H(x(\xi, t), t)$ with respect to $t$ and $\xi$ we get $\bar{H}_{t}=H_{x} v+H_{t}$ and $\bar{H}_{\xi}=H_{x} x_{\xi}$. Then

$$
\begin{aligned}
\left\|\bar{H}_{t}\right\|_{1, \Omega}^{2} & \leq c\left(\left\|H_{x}(x(\xi, t), t) v(x(\xi, t), t)\right\|_{1, \Omega}^{2}+\alpha(a)\left\|H_{t}\right\|_{1, \Omega_{t}}^{2}\right) \\
& \leq c\left(\left\|H_{x}(x(\xi, t), t)\right\|_{1, \Omega}^{2}\|v(x(\xi, t), t)\|_{2, \Omega}^{2}+\alpha(a)\left\|H_{t}\right\|_{1, \Omega_{t}}^{2}\right) \\
& \leq c \gamma\left(t \int_{0}^{t}\|v\|_{3, \Omega_{\tau}}^{2} d \tau\right)\left(\|H\|_{2, \Omega_{t}}^{2}\|v\|_{2, \Omega_{t}}^{2}+\left\|H_{t}\right\|_{1, \Omega_{t}}^{2}\right) .
\end{aligned}
$$

Hence the first inequality is proved. Similarly we can show the other inequalities.

In Lemmas 3.7, 3.8, 3.10 and 3.11 we need

Lemma 2.2. For a solution of problem (1.1)-(1.7) we have

$$
\left\|\bar{v}_{t}\right\|_{1, \Omega}^{2} \geq c \gamma\left(t \int_{0}^{t}\|v\|_{3, \Omega_{\tau}}^{2} d \tau\right)\left(\left\|v_{t}\right\|_{1, \Omega_{t}}^{2}-\|v\|_{2, \Omega_{t}}^{2}\|v\|_{3, \Omega_{t}}^{2}\right),
$$

where $\gamma$ is a positive function.

Proof. From $\bar{v}_{t}=v_{x} v+v_{t}$ we get $\int_{\Omega} \bar{v}_{t}^{2} d \xi=\int_{\Omega} v_{t}^{2} d \xi+2 \int_{\Omega} v_{t} \cdot v_{x} v d \xi$ $+\int_{\Omega}\left(v_{x} v\right)^{2} d \xi$ and from $\bar{v}_{t \xi}=v_{t x} x_{\xi}+v_{x x} v x_{\xi}+v_{x}^{2} x_{\xi}$ we get

$$
\int_{\Omega} \bar{v}_{t \xi}^{2} d \xi=\int_{\Omega}\left(v_{t x} x_{\xi}\right)^{2} d \xi+2 \int_{\Omega}\left(v_{t x} x_{\xi}\right) b d \xi+\int_{\Omega} b^{2} d \xi,
$$

where $b=v_{x x} v x_{\xi}+v_{x}^{2} x_{\xi}$. Hence we obtain (2.8).

Similarly we obtain an inequality for

$$
\|\bar{v}\|_{i, \Omega}^{2} ;\|\bar{H}\|_{i, \Omega}^{2}, \quad i=1,2,3 ; \quad\|\bar{p}\|_{2, \Omega}^{2} ;\left\|\bar{v}_{t}\right\|_{2, \Omega}^{2} ;\left\|\bar{p}_{t}\right\|_{1, \Omega}^{2} ;\left\|\bar{H}_{t}\right\|_{2, \Omega}^{2} .
$$

In Lemmas 3.10, 3.11 we use inequalities (3.16), (3.19), (3.20) in local coordinates $z$, connected with $\{\xi\}$ (see [1]). 
3. Differential inequality. Assume that the existence of a sufficiently smooth local solution of problem (1.1)-(1.7) has been proved and

(*) $\stackrel{2}{H}_{\mid B}=0 \quad$ on $B ; \quad \mathbb{T}\left(v, p^{\prime}\right) n=\left(-\mu_{1} \stackrel{1}{H} \otimes \stackrel{1}{H}+\mu_{1} \frac{\stackrel{1}{H}^{2}}{2} I\right) n \quad$ on $\widetilde{S}^{T}$,

where $p^{\prime}=p-p_{0}, f=0, \int_{\Omega} v_{0} d x=0, \int_{\Omega} v_{0} \cdot \varphi_{i} d x=0$, and $\varphi_{i}, i=1,2,3$, are defined in Lemma 4.1.

In this section we obtain a special differential inequality which enables us to prove the existence of a global solution.

REMARK 3.1. Integrating $(1.1)_{1}$ over $\Omega_{t}$ we get

$\frac{d}{d t} \int_{\Omega_{t}} v d x-\int_{\Omega_{t}} \operatorname{div} \mathbb{T}\left(v, p^{\prime}\right) d x+\mu_{1} \int_{\Omega_{t}}\left(-\operatorname{div}(\stackrel{1}{H} \otimes \stackrel{1}{H})+\nabla \frac{H^{2}}{2} I\right) d x=\int_{\Omega_{t}} f d x$.

Then from $(*)$ we get

$$
\begin{aligned}
\frac{d}{d t} \int_{\Omega_{t}} v d x+\int_{S_{t}}\left(\mu_{1} \stackrel{1}{H} \otimes \stackrel{1}{H}\right. & \left.-\mu_{1} \frac{\stackrel{1}{H}}{2} I\right) n d x_{S_{t}} \\
& +\mu_{1} \int_{\Omega_{t}}\left(-\operatorname{div}(\stackrel{1}{H} \otimes \stackrel{1}{H})+\nabla \frac{\stackrel{1}{H}^{2}}{2}\right) d x=0 .
\end{aligned}
$$

Integrating the last equality by parts we get

$$
\int_{\Omega_{t}} v d x=\int_{\Omega} v_{0} d x=0
$$

REMARK 3.2. Let $\varphi_{i}, i=1,2,3$, be defined in Lemma 4.1. Multiplying $(1.1)_{1}$ by $\varphi_{i}, i=1,2,3$, and integrating over $\Omega_{t}$ we get

$$
\begin{aligned}
\frac{d}{d t} \int_{\Omega_{t}} v \cdot \varphi_{i} d x & -\int_{\Omega_{t}} \operatorname{div}\left(\mathbb{T}\left(v, p^{\prime}\right) \varphi_{i}\right) d x \\
& +\mu_{1} \int_{\Omega_{t}}\left(-\operatorname{div}\left(\stackrel{1}{H} \otimes \stackrel{1}{H} \varphi_{i}\right)+\nabla \frac{\stackrel{H}{H}^{2}}{2} \varphi_{i}\right) d x=\int_{\Omega_{t}} f \cdot \varphi_{i} d x
\end{aligned}
$$

Then from $(*)$ we get

$$
\begin{aligned}
\frac{d}{d t} \int_{\Omega_{t}} v \varphi_{i} d x+\int_{S_{t}} & \left(\mu_{1} \stackrel{1}{H} \otimes \stackrel{1}{H}-\mu_{1} \frac{H^{2}}{2} I\right) \varphi_{i} \cdot n d x_{S_{t}} \\
& +\mu_{1} \int_{\Omega_{t}}\left(-\operatorname{div}(\stackrel{1}{H} \otimes \stackrel{1}{H}) \cdot \varphi_{i}+\nabla \frac{\stackrel{H}{H}^{2}}{2} \cdot \varphi_{i}\right) d x_{S_{t}}=0 .
\end{aligned}
$$


Integrating the last equality by parts we get

$$
\int_{\Omega_{t}} v \cdot \varphi_{i} d x=\int_{\Omega} v_{0} \cdot \varphi_{i} d x=0, \quad i=1,2,3 .
$$

LEMMA 3.1. For a sufficiently smooth solution $\left(v, p^{\prime}, H\right)$ of (1.1)-(1.7), we have

$$
\frac{d}{d t}\|v\|_{0, \Omega_{t}}^{2}+\|v\|_{1, \Omega_{t}}^{2} \leq c\|\stackrel{1}{H}\|_{1, \Omega_{t}}^{4} .
$$

Proof. Multiplying $(1.1)_{1}$ by $v$ and integrating over $\Omega_{t}$ we get

$$
\begin{array}{r}
\frac{1}{2} \int_{\Omega_{t}} \partial_{t} v^{2} d x+\int_{\Omega_{t}} v \cdot \nabla v \cdot v d x+\int_{\Omega_{t}} \mathbb{D}^{2}(v) d x-\mu_{1} \int_{\Omega_{t}} \stackrel{1}{H} \cdot \nabla \stackrel{1}{H} v d x \\
\quad+\mu_{1} \int_{\Omega_{t}} \nabla \frac{\stackrel{1}{H}^{2}}{2} v d x+\mu_{1} \int_{S_{t}}\left(\stackrel{1}{H} \otimes \stackrel{1}{H}-\frac{\stackrel{1}{H}^{2}}{2} I\right) v n d x_{S_{t}}=0 .
\end{array}
$$

Using

$$
\int_{\Omega_{t}} v \cdot v_{t} d x=\frac{1}{2} \frac{d}{d t} \int_{\Omega_{t}} v^{2} d x-\int_{\Omega_{t}} v \cdot \nabla v v d x
$$

and Lemma 4.1 we get (3.1).

LEMMA 3.2. For a sufficiently smooth solution $\left(v, p^{\prime}, H\right)$ of (1.1)-(1.7), we have

$$
\begin{aligned}
\frac{d}{d t}\left\|v_{t}\right\|_{0, \Omega_{t}}^{2}+\left\|v_{t}\right\|_{1, \Omega_{t}}^{2} \leq & c\left[\left\|v_{t}\right\|_{1, \Omega_{t}}^{2}\|v\|_{1, \Omega_{t}}^{2}+\left\|\stackrel{1}{H_{t}}\right\|_{1, \Omega_{t}}^{2}\|\stackrel{1}{H}\|_{1, \Omega_{t}}^{2}\right. \\
& \left.+\left(\|v\|_{1, \Omega_{t}}^{2}+\|H\|_{1, \Omega_{t}}^{2}\right)^{2}\right] \equiv X_{1} .
\end{aligned}
$$

Proof. Differentiating $(1.1)_{1}$ with respect to $t$, multiplying by $v_{t}$ and integrating over $\Omega_{t}$ we get

$$
\begin{aligned}
& \frac{1}{2} \int_{\Omega_{t}}\left(v_{t}\right)_{t}^{2} d x+\int_{\Omega_{t}} v_{t} \cdot \nabla v \cdot v_{t} d x+\int_{\Omega_{t}} v \cdot \nabla v_{t} \cdot v_{t} d x \\
& +\int_{\Omega_{t}} \mathbb{D}^{2}\left(v_{t}\right) d x-\mu_{1} \int_{\Omega_{t}}(\stackrel{1}{H} \cdot \nabla \stackrel{1}{H})_{t} v_{t} d x+\mu_{1} \int_{\Omega_{t}}\left(\nabla \stackrel{1}{H}^{2}\right)_{t} v_{t} d x \\
& +\mu_{1} \int_{S_{t}}\left[\left(\stackrel{1}{H} \otimes \stackrel{1}{H}-\frac{\stackrel{H}{H}^{2}}{2} I\right) n\right]_{t} v_{t} d x_{S_{t}}=0 .
\end{aligned}
$$

Using

$$
\int_{\Omega_{t}} v_{t} \cdot v_{t t} d x=\frac{1}{2} \frac{d}{d t} \int_{\Omega_{t}} v_{t}^{2} d x-\int_{\Omega_{t}} v_{t} \cdot \nabla v_{t} v d x
$$

and Lemma 4.2 we get (3.3). 
LEMMA 3.3. For a sufficiently smooth solution $\left(v, p^{\prime}, H\right)$ of (1.1)-(1.7), we have

$$
\begin{aligned}
\frac{d}{d t}\left\|v_{t t}\right\|_{0, \Omega_{t}}^{2}+\left\|v_{t t}\right\|_{1, \Omega_{t}}^{2} \leq & c\left[\left\|v_{t t}\right\|_{1, \Omega_{t}}^{2}\|v\|_{2, \Omega_{t}}^{2}+\left\|v_{t}\right\|_{1, \Omega_{t}}^{4}\right. \\
& +\left\|\stackrel{1}{H_{t t}}\right\|_{0, \Omega_{t}}^{2}\|\stackrel{1}{H}\|_{1, \Omega_{t}}^{2}+\left\|\stackrel{1}{H_{t}}\right\|_{1, \Omega_{t}}^{4} \\
& +\left(\left\|v_{t}\right\|_{1, \Omega_{t}}^{2}+\|v\|_{2, \Omega_{t}}^{2}\left(\|v\|_{1, \Omega_{t}}^{2}+1\right)\right. \\
& \left.\left.+\left\|\stackrel{1}{H}_{t}\right\|_{1, \Omega_{t}}^{2}+\|\stackrel{1}{H}\|_{1, \Omega_{t}}^{2}\right)^{2}\right] \equiv X_{2}
\end{aligned}
$$

Proof. Differentiating $(1.1)_{1}$ twice with respect to $t$, multiplying by $v_{t t}$, integrating over $\Omega_{t}$ and using the equality

$$
\int_{\Omega_{t}} v_{t t} \cdot v_{t t t} d x=\frac{1}{2} \frac{d}{d t} \int_{\Omega_{t}}\left(v_{t t}\right)^{2} d x-\int_{\Omega_{t}} v_{t t} \cdot \nabla v_{t t} v d x
$$

and Lemma 4.3, we get (3.5).

LEMMA 3.4. For a sufficiently smooth solution $\left(v, p^{\prime}, H\right)$ of $(1.1)-(1.7)$, we have

$$
\frac{d}{d t}\|H\|_{0, \Pi_{t}}^{2}+\|H\|_{1, \Pi_{t}}^{2} \leq c\|H\|_{1, \Pi_{t}}^{2}\|v\|_{1, \Pi_{t}}^{2} .
$$

Proof. Multiplying (1.1) $)_{3,4}$ by $H$ and integrating over $\Pi_{t}$, and using the equality

$$
\int_{\Pi_{t}} H \cdot H_{t} d x=\frac{1}{2} \frac{d}{d t} \int_{\Pi_{t}} H^{2} d x-\int_{\Pi_{t}} H \cdot \nabla H v d x
$$

we get (3.6).

LEMMA 3.5. For a sufficiently smooth solution $\left(v, p^{\prime}, H\right)$ of $(1.1)-(1.7)$, we have

$$
\frac{d}{d t}\left\|H_{t}\right\|_{0, \Pi_{t}}^{2}+\left\|H_{t}\right\|_{1, \Pi_{t}}^{2} \leq c\left(\left\|H_{t}\right\|_{1, \Pi_{t}}^{2}\|v\|_{1, \Pi_{t}}^{2}+\left\|v_{t}\right\|_{1, \Pi_{t}}^{2}\|H\|_{1, \Pi_{t}}^{2}\right) .
$$

Proof. Differentiating $(1.1)_{3,4}$ with respect to $t$, multiplying by $H_{t}$, integrating over $\Pi_{t}$ and using the equality

$$
\int_{\Pi_{t}} H_{t} \cdot H_{t t} d x=\frac{1}{2} \frac{d}{d t} \int_{\Pi_{t}}\left(H_{t}\right)^{2} d x-\int_{\Pi_{t}} H_{t} \cdot \nabla H_{t} v d x
$$

we get $(3.7)$.

LEMMA 3.6. For a sufficiently smooth solution $\left(v, p^{\prime}, H\right)$ of (1.1)-(1.7), we have

$$
\begin{aligned}
\frac{d}{d t}\left\|H_{t t}\right\|_{0, \Pi_{t}}^{2}+\left\|H_{t t}\right\|_{1, \Pi_{t}}^{2} \leq & c\left(\left\|H_{t t}\right\|_{1, \Pi_{t}}^{2}\|v\|_{1, \Pi_{t}}^{2}+\left\|v_{t t}\right\|_{1, \Pi_{t}}^{2}\|H\|_{1, \Pi_{t}}^{2}\right) \\
& +\left\|v_{t}\right\|_{1, \Pi_{t}}^{2}\left\|H_{t}\right\|_{1, \Pi_{t}}^{2} \equiv X_{3} .
\end{aligned}
$$


Proof. Differentiating (1.1) $)_{3,4}$ twice with respect to $t$, multiplying by $H_{t t}$, integrating over $\Pi_{t}$ and using the equality

$$
\int_{\Pi_{t}} H_{t t} \cdot H_{t t t} d x=\frac{1}{2} \frac{d}{d t} \int_{\Pi_{t}}\left(H_{t t}\right)^{2} d x-\int_{\Pi_{t}} H_{t t} \cdot \nabla H_{t t} v d x
$$

we get (3.8).

Lemma 3.7. For a sufficiently smooth solution $\left(v, p^{\prime}, H\right)$ of (1.1)-(1.7), we have

$$
\begin{aligned}
\frac{d}{d t}\left\|v_{t}\right\|_{0, \Omega_{t}}^{2} & +\frac{d}{d t}\|v\|_{2, \Omega_{t}}^{2}+\|v\|_{3, \Omega_{t}}^{2}+\left\|p^{\prime}\right\|_{2, \Omega_{t}}^{2} \\
\leq & c\left[\gamma\left(t \int_{0}^{t}\|v\|_{3, \Omega_{\tau}}^{2} d \tau\right)\|\stackrel{1}{H}\|_{1, \Omega_{t}}^{2}\|\stackrel{1}{H}\|_{3, \Omega_{t}}^{2}\right. \\
& \left.+\varepsilon\left(\left\|v_{t}\right\|_{2, \Omega_{t}}^{2}+\|v\|_{3, \Omega_{t}}^{2}\right)+\|v\|_{3, \Omega_{t}}^{2}\|v\|_{1, \Omega_{t}}^{2}+X_{1}\right] \equiv X_{4}
\end{aligned}
$$

Proof. Similarly to [1] we prove the inequality

$$
\begin{aligned}
\|\bar{v}\|_{3, \Omega}^{2}+\left\|\bar{p}^{\prime}\right\|_{2, \Omega}^{2} \leq & \alpha(a)\left(\left\|\frac{1}{\bar{H}}\right\|_{2, \Omega}^{2}\left\|\frac{1}{\bar{H}}\right\|_{3, \Omega}^{2}+\left\|\bar{v}_{t}\right\|_{1, \Omega}^{2}\right. \\
& \left.+\left\|\left(-\mu_{1} \frac{1}{H} \otimes \frac{1}{H}+\mu_{1} \frac{\frac{1}{H}^{2}}{2} I\right) \bar{n}_{v}\right\|_{3 / 2, S}^{2}+\|\bar{v}\|_{0, \Omega}^{2}\right) .
\end{aligned}
$$

Then from

$$
\frac{d}{d t}\|v\|_{2, \Omega_{t}}^{2} \leq c\left(\|v\|_{2, \Omega_{t}}^{2}+\varepsilon\left\|v_{t}\right\|_{2, \Omega_{t}}^{2}+\|v\|_{3, \Omega_{t}}^{2}\|v\|_{1, \Omega_{t}}^{2}\right)
$$

and (3.3) we get (3.9).

Lemma 3.8. For a sufficiently smooth solution $\left(v, p^{\prime}, H\right)$ of (1.1)-(1.7), we have

$$
\begin{aligned}
& \frac{d}{d t}\left\|v_{t}\right\|_{1, \Omega_{t}}^{2}+\frac{d}{d t}\left\|v_{t t}\right\|_{0, \Omega_{t}}^{2}+\frac{d}{d t}\left\|v_{t}\right\|_{0, \Omega_{t}}^{2}+\left\|v_{t}\right\|_{2, \Omega_{t}}^{2}+\left\|p_{t}^{\prime}\right\|_{1, \Omega_{t}}^{2} \\
& \leq c\left[\gamma ( t \int _ { 0 } ^ { t } \| v \| _ { 3 , \Omega _ { \tau } } ^ { 2 } d \tau ) \left(\|v\|_{2, \Omega_{t}}^{2}\left(\|v\|_{3, \Omega_{t}}^{2}+\left\|p^{\prime}\right\|_{2, \Omega_{t}}^{2}+\|H\|_{2, \Omega_{t}}^{4}\right)\right.\right. \\
& \left.\quad+\left\|H_{t}^{1}\right\|_{1, \Omega_{t}}^{2}\|\stackrel{1}{H}\|_{2, \Omega_{t}}^{2}+\left\|H_{t}\right\|_{2, \Omega_{t}}^{2}\|\stackrel{1}{H}\|_{1, \Omega_{t}}^{2}\right) \\
& \left.\quad+\varepsilon\left(\left\|v_{t t}\right\|_{1, \Omega_{t}}^{2}+\left\|v_{t}\right\|_{2, \Omega_{t}}^{2}\right)+\left\|v_{t}\right\|_{2, \Omega_{t}}^{2}\|v\|_{1, \Omega_{t}}^{2}+X_{1}+X_{2}\right] .
\end{aligned}
$$


Proof. Similarly to [1] we prove the inequality

$$
\begin{aligned}
\left\|\bar{v}_{t}\right\|_{2, \Omega}^{2}+\left\|\bar{p}_{t}^{\prime}\right\|_{1, \Omega}^{2} & \\
\leq & \alpha(a)\left[\|\bar{v}\|_{2, \Omega}^{2}\left(\|\bar{v}\|_{3, \Omega}^{2}+\left\|\bar{p}^{\prime}\right\|_{2, \Omega}^{2}+\left\|\frac{1}{H}\right\|_{2, \Omega}^{4}\right)\right. \\
& +\left\|\bar{H}_{t}\right\|_{2, \Omega}^{2}\left\|\frac{1}{H}\right\|_{2, \Omega}^{2}+\left\|\bar{H}_{t}\right\|_{2, \Omega}^{2}\left\|\frac{1}{H}\right\|_{2, \Omega}^{2}+\left\|\bar{v}_{t t}\right\|_{0, \Omega}^{2} \\
& +\left\|\left[\left(-\mu_{1} \frac{1}{H} \otimes \frac{1}{H}-\mu_{1} \frac{\frac{1}{H}^{2}}{2} I\right) \bar{n}_{v}\right]\right\|_{t}^{2} \|_{1 / 2, S} \\
& \left.+\left\|\left(\bar{n}_{v} \mathbb{T}_{v}\right)_{t}\left(\bar{v}, \bar{p}^{\prime}\right)\right\|_{1 / 2, S}^{2}+\left\|\bar{v}_{t}\right\|_{0, \Omega}^{2}\right]
\end{aligned}
$$

Then from

$$
\frac{d}{d t}\left\|v_{t}\right\|_{1, \Omega_{t}}^{2} \leq\left\|v_{t}\right\|_{1, \Omega_{t}}^{2}+\varepsilon\left\|v_{t t}\right\|_{1, \Omega_{t}}^{2}+\left\|v_{t}\right\|_{2, \Omega_{t}}^{2}\|v\|_{1, \Omega_{t}}^{2}
$$

and (3.3), (3.5) we get (3.11).

Lemma 3.9. For a sufficiently smooth solution $\left(v, p^{\prime}, H\right)$ of (1.1)-(1.7), we have

$$
\begin{aligned}
\frac{d}{d t}\|v\|_{1, \Omega_{t}}^{2}+ & \frac{d}{d t}\left\|v_{t}\right\|_{0, \Omega_{t}}^{2}+\|v\|_{2, \Omega_{t}}^{2} \\
\leq & c\left[\gamma\left(t \int_{0}^{t}\|v\|_{3, \Omega_{\tau}}^{2} d \tau\right)\|H\|_{2, \Omega_{t}}^{4}\right. \\
& \left.+\varepsilon\left(\left\|v_{t}\right\|_{1, \Omega_{t}}^{2}+\|v\|_{2, \Omega_{t}}^{2}\right)+\|v\|_{2, \Omega_{t}}^{2}\|v\|_{0, \Omega_{t}}^{2}+X_{1}\right] .
\end{aligned}
$$

Proof. Similarly to [1] we prove the inequality

$$
\begin{aligned}
\|\bar{v}\|_{2, \Omega}^{2} \leq & \alpha(a)\left[\left\|\frac{1}{\bar{H}}\right\|_{1, \Omega}^{4}+\left\|\frac{1}{\bar{H}}\right\|_{0, \Omega}^{2}\left\|\frac{1}{\bar{H}}\right\|_{2, \Omega}^{2}+\left\|\bar{v}_{t}\right\|_{0, \Omega}^{2}\right. \\
& \left.+\left\|\left(-\mu_{1} \frac{1}{H} \otimes \frac{1}{H}+\mu_{1} \frac{\frac{1}{H}^{2}}{2} I\right) \bar{n}_{v}\right\|_{1 / 2, S}^{2}+\|\bar{v}\|_{0, \Omega}^{2}\right] .
\end{aligned}
$$

Then from

$$
\frac{d}{d t}\|v\|_{1, \Omega_{t}}^{2} \leq c\left(\|v\|_{1, \Omega_{t}}^{2}+\varepsilon\left\|v_{t}\right\|_{1, \Omega_{t}}^{2}+\|v\|_{2, \Omega_{t}}^{2}\|v\|_{0, \Omega_{t}}^{2}\right)
$$

and (3.3), we get (3.13).

LEMMA 3.10. For a sufficiently smooth solution $\left(v, p^{\prime}, H\right)$ of (1.1)-(1.7), we have

$$
\frac{d}{d t}\left\|H_{t}\right\|_{2, \Pi_{t}}^{2}+\frac{d}{d t}\left\|H_{t t}\right\|_{0, \Pi_{t}}^{2}+\frac{d}{d t}\left\|v_{t}\right\|_{0, \Omega_{t}}^{2}+\frac{d}{d t}\|v\|_{2, \Omega_{t}}^{2}+\left\|H_{t}\right\|_{2, \Pi_{t}}^{2}
$$




$$
\begin{aligned}
\leq & c \gamma\left(t \int_{0}^{t}\|v\|_{3, \Omega_{\tau}}^{2} d \tau\right)\left[\left\|H_{t}\right\|_{2, \Pi_{t}}^{2}\|v\|_{1, \Pi_{t}}^{2}+\|H\|_{2, \Pi_{t}}^{2}\right. \\
& \cdot\left(\|v\|_{3, \Pi_{t}}^{2}+\left\|v_{t}\right\|_{2, \Pi_{t}}^{2}+\|v\|_{2, \Pi_{t}}^{4}\right) \\
& +\|v\|_{3, \Pi_{t}}^{2}\left(\left\|H_{t}\right\|_{1, \Pi_{t}}^{2}+\|H\|_{2, \Pi_{t}}^{2}\|v\|_{2, \Pi_{t}}^{2}+\|v\|_{2, \Pi_{t}}^{2}+\left\|v_{t}\right\|_{1, \Pi_{t}}^{2}\right) \\
& \left.+a^{2}\|H\|_{3, \Pi_{t}}^{2}+X_{3}+X_{4}\right] \equiv X_{5} .
\end{aligned}
$$

Proof. From the inequalities (see [1])

$$
\begin{aligned}
\frac{d}{d t}\left\|\widetilde{H}_{t \tau}\right\|_{0, \widehat{\Omega}}^{2}+ & \left\|\widetilde{H}_{t}\right\|_{2, \widehat{\Omega}}^{2} \\
\leq & \alpha(\widehat{a})\left[\left\|\widetilde{H}_{t t}\right\|_{0, \widehat{\Omega}}^{2}+\|\widehat{v}\|_{3, \widehat{\Omega}}^{2}\left(\|\widehat{H}\|_{1, \widehat{\Omega}}^{2}+\left\|\widehat{H}_{t}\right\|_{1, \widehat{\Omega}}^{2}\right.\right. \\
& \left.+\left\|\widehat{v}_{t}\right\|_{1, \widehat{\Omega}}^{2}+\|\widehat{H}\|_{2, \widehat{\Omega}}^{2}\|\widehat{v}\|_{2, \widehat{\Omega}}^{2}\|\widehat{v}\|_{2, \widehat{\Omega}}^{2}+1\right) \\
& \left.+\left\|\widehat{v}_{t}\right\|_{2, \widehat{\Omega}}^{2}+\|\widehat{H}\|_{2, \widehat{\Omega}}^{2}\left\|\widehat{v}_{t}\right\|_{2, \widehat{\Omega}}^{2}\right], \\
\frac{d}{d t}\left\|\widetilde{H}_{\tau t}\right\|_{0, \widehat{\Omega}}^{2} \leq & c\left(\varepsilon\left\|\widehat{H}_{t}\right\|_{1, \widehat{\Omega}}^{2}+\left\|\widehat{H}_{t t}\right\|_{1, \widehat{\Omega}}^{2}\right), \\
\frac{d}{d t}\left\|H_{t}\right\|_{1, \Pi_{t}}^{2} \leq & c\left(\varepsilon\left\|H_{t}\right\|_{1, \Pi_{t}}^{2}+\left\|H_{t t}\right\|_{1, \Pi_{t}}^{2}+\left\|H_{t}\right\|_{2, \Pi_{t}}^{2}\|v\|_{1, \Pi_{t}}^{2}\right),
\end{aligned}
$$

we get

$$
\begin{aligned}
& \frac{d}{d t}\left\|H_{t}\right\|_{1, \Pi_{t}}^{2}+\left\|H_{t}\right\|_{2, \Pi_{t}}^{2} \\
& \leq \gamma\left(t \int_{0}^{t}\|v\|_{3, \Omega_{\tau}}^{2} d \tau\right)\left[\left\|H_{t}\right\|_{1, \Pi_{t}}^{2}+\left\|H_{t}\right\|_{2, \Pi_{t}}^{2}\|v\|_{1, \Pi_{t}}^{2}\right. \\
& \quad+\|H\|_{2, \Pi_{t}}^{2}\left(\|v\|_{3, \Pi_{t}}^{2}+\left\|v_{t}\right\|_{2, \Pi_{t}}^{2}+\|v\|_{2, \Pi_{t}}^{4}\right)+\|v\|_{3, \Pi_{t}}^{2}\left(\left\|H_{t}\right\|_{1, \Pi_{t}}^{2}\right. \\
& \left.\left.\quad+\|H\|_{2, \Pi_{t}}^{2}\|v\|_{2, \Pi_{t}}^{2}+\|v\|_{2, \Pi_{t}}^{2}+\left\|v_{t}\right\|_{2, \Pi_{t}}^{2}\right)+\|v\|_{2, \Pi_{t}}^{2}+a^{2}\|H\|_{3, \Pi_{t}}^{2}\right] .
\end{aligned}
$$

Using (3.8), (3.9), we get (3.15).

LEMMA 3.11. For a sufficiently smooth solution $\left(v, p^{\prime}, H\right)$ of (1.1)-(1.7), we have

$$
\begin{gathered}
\frac{d}{d t}\|H\|_{2, \Pi_{t}}^{2}+\frac{d}{d t}\left\|H_{t t}\right\|_{0, \Pi_{t}}^{2}+\frac{d}{d t}\left\|v_{t}\right\|_{0, \Omega_{t}}^{2}+\frac{d}{d t}\|v\|_{2, \Omega_{t}}^{2}+\left\|H_{t}\right\|_{2, \Pi_{t}}^{2}+\|H\|_{3, \Pi_{t}}^{2} \\
\leq c \gamma\left(t \int_{0}^{t}\|v\|_{3, \Omega_{\tau}}^{2} d \tau\right)\left[\|H\|_{2, \Pi_{t}}^{2}\|v\|_{2, \Pi_{t}}^{2}+\|H\|_{2, \Pi_{t}}^{2}\|v\|_{3, \Pi_{t}}^{2}\right. \\
\left.+\|H\|_{3, \Pi_{t}}^{2}\|v\|_{2, \Pi_{t}}^{2}+X_{4}+X_{5}\right] \equiv X_{6}
\end{gathered}
$$

Proof. From the inequalities (see [1])

$$
\begin{aligned}
\frac{d}{d t}\left\|\widetilde{H}_{\tau}\right\|_{0, \widehat{\Omega}}^{2}+\|\widetilde{H}\|_{2, \widehat{\Omega}}^{2} \leq & \alpha_{1}(\widehat{a})\left[\|\widehat{H}\|_{1, \widehat{\Omega}}^{2}\|\widehat{v}\|_{2, \widehat{\Omega}}^{2}+\|\widehat{v}\|_{2, \widehat{\Omega}}^{2}+\|\widehat{H}\|_{1, \widehat{\Omega}}^{2}\right. \\
& \left.+\left\|\widehat{H}_{t}\right\|_{0, \widehat{\Omega}}^{2}+\widehat{a}^{2}\|\widehat{H}\|_{3, \widehat{\Omega}}^{2}\right],
\end{aligned}
$$




$$
\begin{aligned}
\frac{d}{d t}\left\|\widetilde{H}_{\tau \tau}\right\|_{0, \widehat{\Omega}}^{2}+\|\widetilde{H}\|_{3, \widehat{\Omega}}^{2} \leq & \alpha_{2}(\widehat{a})\left[\|\widehat{H}\|_{2, \widehat{\Omega}}^{2}\|\widehat{v}\|_{2, \widehat{\Omega}}^{2}+\|\widehat{v}\|_{2, \widehat{\Omega}}^{2}\right. \\
& \left.+\|\widehat{H}\|_{2, \widehat{\Omega}}^{2}+\|\widehat{H}\|_{1, \widehat{\Omega}}^{2}\|\widehat{v}\|_{3, \widehat{\Omega}}^{2}+\left\|\widehat{H}_{t}\right\|_{1, \widehat{\Omega}}^{2}\right],
\end{aligned}
$$

$$
\begin{aligned}
& \frac{d}{d t}\left\|\widetilde{H}_{\tau}\right\|_{0, \widehat{\Omega}}^{2} \leq c\left(\varepsilon\|\widehat{H}\|_{1, \widehat{\Omega}}^{2}+\left\|\widehat{H}_{t}\right\|_{1, \widehat{\Omega}}^{2}\right), \\
& \frac{d}{d t}\left\|\widetilde{H}_{\tau \tau}\right\|_{0, \widehat{\Omega}}^{2} \leq c\left(\varepsilon\|\widehat{H}\|_{2, \widehat{\Omega}}^{2}+\left\|\widehat{H}_{t}\right\|_{2, \widehat{\Omega}}^{2}\right),
\end{aligned}
$$

we get

$$
\begin{aligned}
\|H\|_{3, \Pi_{t}}^{2} \leq & c \gamma\left(t \int_{0}^{t}\|v\|_{3, \Omega_{\tau}}^{2} d \tau\right)\left[\|H\|_{2, \Pi_{t}}^{2}\|v\|_{2, \Pi_{t}}^{2}+\|v\|_{2, \Pi_{t}}^{2}\right. \\
& \left.+\|H\|_{2, \Pi_{t}}^{2}\|v\|_{3, \Pi_{t}}^{2}+\left\|H_{t}\right\|_{2, \Pi_{t}}^{2}+\|H\|_{3, \Pi_{t}}^{2}\|v\|_{2, \Pi_{t}}^{2}\right] .
\end{aligned}
$$

Then from the inequality

$$
\frac{d}{d t}\|H\|_{2, \Pi_{t}}^{2} \leq \varepsilon\|H\|_{2, \Pi_{t}}^{2}+\left\|H_{t}\right\|_{2, \Pi_{t}}^{2}+\|H\|_{3, \Pi_{t}}^{2}\|v\|_{1, \Pi_{t}}^{2}
$$

and (3.9), (3.15) we get (3.18).

LEMma 3.12. For a sufficiently smooth solution $\left(v, p^{\prime}, H\right)$ of (1.1)-(1.7), we have

$$
\begin{aligned}
\frac{d}{d t}\|H\|_{1, \Pi_{t}}^{2}+\frac{d}{d t}\|H\|_{2, \Pi_{t}}^{2} & +\frac{d}{d t}\left\|H_{t t}\right\|_{0, \Pi_{t}}^{2}+\frac{d}{d t}\left\|v_{t}\right\|_{0, \Omega_{t}}^{2} \\
& +\frac{d}{d t}\|v\|_{2, \Omega_{t}}^{2}+\left\|H_{t}\right\|_{2, \Pi_{t}}^{2}+\|H\|_{3, \Pi_{t}}^{2} \leq X_{6} .
\end{aligned}
$$

Proof. From the inequality

$$
\frac{d}{d t}\|H\|_{1, \Pi_{t}}^{2} \leq\|H\|_{1, \Pi_{t}}^{2}+\varepsilon\left\|H_{t}\right\|_{1, \Pi_{t}}^{2}+\|H\|_{2, \Pi_{t}}^{2}\|v\|_{0, \Pi_{t}}^{2}
$$

and from (3.18) we get (3.22).

Now let $\stackrel{2}{H}=H_{*}$ on $B$; then from Lemmas $3.1-3.12$ we get

Lemma 3.13. For a sufficiently smooth solution $\left(v, p^{\prime}, H\right)$ of (1.1)-(1.7), we have

$$
\begin{aligned}
\frac{d}{d t} \varphi+\phi \leq c & {\left[\left(\gamma\left(t \int_{0}^{t}\|v\|_{3, \Omega_{\tau}}^{2} d \tau\right)+1\right) \phi \varphi(1+\varphi)+\left\|H_{*}\right\|_{3, B}^{2}\right.} \\
& \left.+\left\|H_{*}\right\|_{1, B}^{4}+\left\|H_{* t}\right\|_{2, B}^{2}\left(1+\left\|H_{* t}\right\|_{2, B}^{2}\right)+\left\|H_{* t t}\right\|_{1, B}^{2}\right],
\end{aligned}
$$


where $\gamma$ is an increasing positive function, $t \in[0, T]$ and

$$
\begin{aligned}
& \varphi(t)=\sum_{i+k \leq 2}\left(\left\|\partial_{t}^{i} v\right\|_{k, \Omega_{t}}^{2}+\left\|\partial_{t}^{i} H\right\|_{k, \Pi_{t}}^{2}\right), \\
& \phi(t)=\sum_{\substack{i+k \leq 3 \\
i \leq 2}}\left(\left\|\partial_{t}^{i} v\right\|_{k, \Omega_{t}}^{2}+\left\|\partial_{t}^{i} H\right\|_{k, \Pi_{t}}^{2}\right)+\left\|p^{\prime}\right\|_{2, \Omega_{t}}^{2}+\left\|p_{t}^{\prime}\right\|_{1, \Omega_{t}}^{2} .
\end{aligned}
$$

\section{Korn inequality}

Lemma 4.1. Let $\Omega_{t} \subset \mathbb{R}^{3}$ be a bounded domain. Let $\left(v, p^{\prime}\right)$ be a solution of $(1.1)_{1},(1.1)_{2},(1.5)_{1}$ and $f=\int_{\Omega} v_{0} d x=\int_{\Omega} v_{0} \cdot \varphi_{i} d x=0$, where $\varphi_{i}$ is defined by (4.4), $i=1,2,3$, and

$$
\mathbb{E}_{\Omega_{t}}\left(v_{t}\right)=\int_{\Omega_{t}}\left(\partial_{x_{i}} v_{j t}+\partial_{x_{j}} v_{i t}\right)^{2} d x<\infty .
$$

Then there exists a constant $c$ such that

$$
\left\|v_{t}\right\|_{1, \Omega_{t}}^{2} \leq c\left(\mathbb{E}_{\Omega_{t}}\left(v_{t}\right)+\|v\|_{1, \Omega_{t}}^{4}\right)
$$

Proof. Introduce a function $u$ by

$$
u=\sum_{i=1}^{3} b_{i} \varphi_{i}(x)+v_{t},
$$

where

$$
\begin{aligned}
\varphi_{i} & =(x-\bar{x}) \times e_{i}, \\
\bar{x} & =\frac{1}{\left|\Omega_{t}\right|}\left(\int_{\Omega_{t}} x_{1} d x, \int_{\Omega_{t}} x_{2} d x, \int_{\Omega_{t}} x_{3} d x\right), \\
e_{i} & =\left(\delta_{i 1}, \delta_{i 2}, \delta_{i 3}\right), \quad i=1,2,3 .
\end{aligned}
$$

Define $b=\left(b_{1}, b_{2}, b_{3}\right)$ by

$$
b=\frac{1}{2\left|\Omega_{t}\right|} \int_{\Omega_{t}} \operatorname{rot} v_{t} d x .
$$

Since $\operatorname{rot} \varphi_{i}=2 e_{i} i=1,2,3$, equations (4.3) and (4.4) imply

$$
\int_{\Omega_{t}} \operatorname{rot} u d x=0 .
$$

From (4.4) we have $\int_{\Omega_{t}} \varphi_{i} d x=0, i=1,2,3$, so

$$
\int_{\Omega_{t}} u d x=\int_{\Omega_{t}} v_{t} d x, \quad \text { and also } \quad \mathbb{E}_{\Omega_{t}}\left(\varphi_{i}\right)=0, \quad i=1,2,3,
$$

hence

$$
\mathbb{E}_{\Omega_{t}}(u)=\mathbb{E}_{\Omega_{t}}\left(v_{t}\right) .
$$


By Theorem 1 of [6] we have

(4.9) $\partial_{x_{j}} w_{i}=\varepsilon_{i k l} \partial_{x_{k}} S_{j l}, \quad i=1,2,3, \quad w=\operatorname{rot} u, \quad S_{i j}=\partial_{x_{i}} u_{j}+\partial_{x_{j}} u_{i}$,

so by (4.6) and Lemma 2.4 of [3] it follows that

$$
\|\operatorname{rot} u\|_{0, \Omega_{t}}^{2} \leq c \sum_{i, j=1}^{3}\left\|S_{i j}\right\|_{0, \Omega_{t}}^{2}=c \mathbb{E}_{\Omega_{t}}(u)=c \mathbb{E}_{\Omega_{t}}\left(v_{t}\right)
$$

Employing the identity

$$
\partial_{x_{j}} u_{i}=\frac{1}{2}\left(\partial_{x_{j}} u_{i}+\partial_{x_{i}} u_{j}\right)+\frac{1}{2}\left(\partial_{x_{j}} u_{i}-\partial_{x_{i}} u_{j}\right)
$$

and (4.10) we have

$$
\|\nabla u\|_{0, \Omega_{t}}^{2} \leq c\left(\mathbb{E}_{\Omega_{t}}(u)+\|\operatorname{rot} u\|_{0, \Omega_{t}}^{2}\right) \leq c \mathbb{E}_{\Omega_{t}}(u)=c \mathbb{E}_{\Omega_{t}}\left(v_{t}\right) .
$$

Using (4.3) we obtain

$$
\left\|\nabla v_{t}\right\|_{0, \Omega_{t}}^{2} \leq c\left(\mathbb{E}_{\Omega_{t}}\left(v_{t}\right)+|b|\right) .
$$

Integrating (1.1) ${ }_{1}$ over $\Omega_{t}$ we get

$$
\int_{\Omega_{t}} v_{t} d x=-\int_{\Omega_{t}} v \cdot \nabla v d x
$$

and multiplying $(1.1)_{1}$ by $\varphi_{i}, i=1,2,3$, and integrating over $\Omega_{t}$, from (4.3) we get systems of equations

$$
\sum_{i=1}^{3} b_{i} \int_{\Omega_{t}} \varphi_{i} \cdot \varphi_{j} d x=\int_{\Omega_{t}} u \cdot \varphi_{j} d x+\int_{\Omega_{t}} v \cdot \nabla v \cdot \varphi_{i} d x, \quad j=1,2,3 .
$$

Since $\operatorname{det} \Gamma \neq 0$, where $\Gamma=\left\{\Gamma_{i j}\right\}, \Gamma_{i j}=\int_{\Omega_{t}} \varphi_{i} \cdot \varphi_{j} d x$, we can calculate $b$ from (4.14), so

$$
|b|^{2} \leq c\left(\|u\|_{0, \Omega_{t}}^{2}+\|v\|_{1, \Omega_{t}}^{4}\right) .
$$

Now by the Poincaré inequality and (4.8), (4.12) we obtain

$$
\begin{aligned}
\|u\|_{0, \Omega_{t}}^{2} & \leq 2\left\|u-\frac{1}{\left|\Omega_{t}\right|} \int_{\Omega_{t}} u d x\right\|_{0, \Omega_{t}}^{2}+2\left\|\frac{1}{\left|\Omega_{t}\right|} \int_{\Omega_{t}} u d x\right\|_{0, \Omega_{t}}^{2} \\
& \leq c\left(\|\nabla u\|_{0, \Omega_{t}}^{2}+\left\|\frac{1}{\left|\Omega_{t}\right|} \int_{\Omega_{t}} v_{t} d x\right\|_{0, \Omega_{t}}^{2}\right) \\
& \leq c\left(\mathbb{E}_{\Omega_{t}}(u)+\|v\|_{1, \Omega_{t}}^{4}\right) \\
& =c\left(\mathbb{E}_{\Omega_{t}}\left(v_{t}\right)+\|v\|_{1, \Omega_{t}}^{4}\right) .
\end{aligned}
$$

From (4.3) we get

$$
\left\|v_{t}\right\|_{0, \Omega_{t}}^{2} \leq c\left(\|u\|_{0, \Omega_{t}}^{2}+|b|^{2}\right)
$$

Then from (4.12), (4.15), (4.16) and (4.17) we get (4.2). 
Lemma 4.2. Let $\Omega_{t} \subset \mathbb{R}^{3}$ be a bounded domain. Let $\left(v, p^{\prime}\right)$ be a solution of $(1.1)_{1},(1.1)_{2},(1.5)_{1}$ and $f=\int_{\Omega} v_{0} d x=\int_{\Omega} v_{0} \cdot \varphi_{i} d x, i=1,2,3$, and

$$
\mathbb{E}_{\Omega_{t}}\left(v_{t t}\right)=\int_{\Omega_{t}}\left(\partial_{x_{i}} v_{j t t}+\partial_{x_{j}} v_{i t t}\right)^{2} d x<\infty .
$$

Then there exists a constant $c$ such that

$$
\left\|v_{t t}\right\|_{1, \Omega_{t}}^{2} \leq c\left[\mathbb{E}_{\Omega_{t}}\left(v_{t}\right)+\left\|v_{t}\right\|_{1, \Omega_{t}}^{2}\|v\|_{1, \Omega_{t}}^{2}+\|v\|_{2, \Omega_{t}}^{4}\left(\|v\|_{1, \Omega_{t}}^{2}+1\right)\right] .
$$

Proof. Let $u=\sum_{i=1}^{3} b_{i} \varphi_{i}(x)+v_{t t}$, where $\varphi_{i}$ are described by (4.4). The rest of the argument is as in Lemma 4.1.

5. Global existence. To prove the global existence we introduce the spaces

$$
\begin{aligned}
& \mathcal{N}(t)=\left\{\left(v, p^{\prime}, H\right): \varphi(t)<\infty\right\}, \\
& \mathcal{M}(t)=\left\{\left(v, p^{\prime}, H\right): \varphi(t)+\int_{0}^{t} \phi(\tau) d \tau<\infty\right\},
\end{aligned}
$$

where $\varphi(t), \phi(t)$ are defined by (3.24). From Theorem 2.1 we get

Lemma 5.1. Assume that $\left(v(0), p^{\prime}(0), H(0)\right) \in \mathcal{N}(0)$ and $\varphi(0)<\varepsilon_{1}$. Then $\left(v(t), p^{\prime}(t), H(t)\right) \in \mathcal{M}(t)$ for $t \leq T$, where $T$ is the time of local existence and

$$
\begin{aligned}
\varphi(t)+\int_{0}^{T} \phi(\tau) d \tau \leq & c \varepsilon_{1}+c \int_{0}^{T}\left(\left\|E_{*}\right\|_{0, B}^{2}+\left\|E_{* t}\right\|_{0, B}^{2}\right. \\
& \left.+\left\|H_{*}\right\|_{3, B}^{2}+\left\|H_{* t}\right\|_{2, B}^{2}+\left\|H_{* t t}\right\|_{0, B}^{2}\right) d t \\
\equiv & c\left(\varepsilon_{1}+\beta\right) .
\end{aligned}
$$

Proof. From the inequalities

$$
\begin{aligned}
\|\bar{v}\|_{2, \Omega}^{2} & \leq c\left(\varepsilon\left\|\bar{v}_{t}\right\|_{2,2,2, \Omega^{t}}^{2}+c(\varepsilon)\|\bar{v}\|_{2,2,2, \Omega^{t}}^{2}+\|\bar{v}(0)\|_{2, \Omega}^{2}\right), \\
\|\bar{H}\|_{2, \Pi}^{2} & \leq c\left(\varepsilon\left\|\bar{H}_{t}\right\|_{2,2,2, \Pi^{t}}^{2}+c(\varepsilon)\|\bar{H}\|_{2,2,2, \Pi^{t}}^{2}+\|\bar{H}(0)\|_{2, \Pi}^{2}\right),
\end{aligned}
$$

and Theorem 2.1 we get (5.1).

Lemma 5.2. Assume that there exists a local solution of (1.1)-(1.7) in $\mathcal{M}(t), 0 \leq t \leq T$, with initial data in $\mathcal{N}(0)$ sufficiently small and

$$
\begin{aligned}
\alpha(t) & =\left\|H_{*}\right\|_{3, B}^{2}+\left\|H_{*}\right\|_{1, B}^{4}+\left\|H_{* t}\right\|_{2, B}^{2}\left(1+\left\|H_{* t}\right\|_{2, B}^{2}\right)+\left\|H_{* t t}\right\|_{1, B}^{2} \\
& \leq e^{-\mu t},
\end{aligned}
$$

for $0 \leq t \leq T$, where $\mu>1 / 2$. Then

$$
\varphi(t) \leq e^{-t / 2}\left(\varphi(0)+\frac{c}{\mu-1 / 2}\right) .
$$


Proof. From (3.23) and (5.2) we get

$$
\frac{d}{d t} \varphi+\phi \leq c\left(\gamma\left(t \int_{0}^{t}\|v\|_{3, \Omega_{\tau}}^{2} d \tau\right)+1\right) \phi \varphi(1+\varphi)+c e^{-\mu t} .
$$

From Lemma 5.1 we have $c\left(\gamma\left(t \int_{0}^{t}\|v\|_{3, \Omega_{\tau}}^{2} d \tau\right)+1\right) \phi \varphi(1+\varphi) \leq \frac{1}{2} \phi$ if $\beta$ and $\varepsilon_{1}$ are sufficiently small. Then from (5.4) we get

$$
\frac{d}{d t} \varphi+\frac{1}{2} \phi \leq c e^{-\mu t}
$$

We have $\varphi \leq \phi$. Then from (5.5),

$$
\frac{d}{d t} \varphi+\frac{1}{2} \varphi \leq c e^{-\mu t}
$$

From (5.6) we get (5.3).

LEMMA 5.3. Let the assumptions of Lemma 5.2 be satisfied and $\varphi(0)$ $<\varepsilon_{1}$. Then $\varphi(T) \leq \varepsilon_{1}$ where $T>0$ is the time of local existence.

Proof. If $T$ and $\mu>0$ are sufficiently large, then from (5.3) we get

$$
\varphi(T) \leq e^{-T / 2}\left(\varphi(0)+\frac{c}{\mu-1 / 2}\right) \leq \varphi(0) .
$$

Now we consider problem (1.1)-(1.7) for $t \in[k T,(k+1) T]$. Then similarly to $(3.23)$ we obtain the inequality

$$
\begin{aligned}
\frac{d}{d t} \varphi+\phi \leq & c\left[\left(\gamma\left((t-k T) \int_{k T}^{t}\|v\|_{3, \Omega_{\tau}}^{2} d \tau\right)+1\right) \phi \varphi(1+\varphi)+\left\|H_{*}\right\|_{3, B}^{2}\right. \\
& \left.+\left\|H_{*}\right\|_{1, B}^{4}+\left\|H_{* t}\right\|_{2, B}^{2}\left(1+\left\|H_{* t}\right\|_{2, B}^{2}\right)+\left\|H_{* t t}\right\|_{1, B}^{2}\right],
\end{aligned}
$$

where $t \in[k T,(k+1) T]$.

Let $\varphi(k T) \leq \varphi(0)$. Then from (5.7) similarly to (5.3) we get, for $t \in$ $[k T,(k+1) T]$,

$$
\varphi(t) \leq c \frac{e^{(1 / 2-\mu) k T-t / 2}}{\mu-1 / 2}+\varphi(k T) e^{(k T-t) / 2} \leq c \frac{e^{-t / 2}}{\mu-1 / 2}+\varphi(0) e^{(k T-t) / 2} .
$$

Therefore if $T, \mu>0$ are sufficiently large we get $\varphi((k+1) T) \leq \varphi(0)$. We also obtain the inequalities

$$
\int_{k T}^{t} \phi d \tau \leq \frac{2 c}{\mu} e^{-\mu k T}+2 \varphi(k T) \quad \text { for } t \in[k T,(k+1) T] .
$$

Hence

$$
\int_{k T}^{(k+1) T}\|v\|_{3, \Omega_{t}}^{2} d t \leq \int_{k T}^{(k+1) T} \phi d t \leq \frac{c}{\mu} e^{-\mu k T}+2 \varphi(k T)
$$


and

$$
\int_{k T}^{t} \varphi(\tau) d \tau \leq \frac{2 c}{\mu-1 / 2} e^{-\mu k T}+2 \varphi(k T) \quad \text { for } t \in[k T,(k+1) T] .
$$

Then inequalities (5.9) and (5.11) imply

$$
\begin{aligned}
\left|\int_{k T}^{(k+1) T} v d t\right| & \leq c T^{1 / 2}\left(\int_{k T}^{(k+1) T}\|v\|_{2, \Omega_{t}}^{2} d t\right)^{1 / 2} \\
& \leq c T^{1 / 2}\left(\frac{2 c}{\mu-1 / 2} e^{-\mu k T}+2 \varphi(k T)\right)^{1 / 2} .
\end{aligned}
$$

Proof of Main Theorem. The theorem is proved step by step using local existence in a fixed time interval. Under the assumptions that

$$
\left(v(0), p^{\prime}(0), H(0)\right) \in \mathcal{N}(0)
$$

Theorem 2.1 and Lemma 5.1 yield local existence of solutions of (1.1)-(1.7).

By (5.13) and Lemma 5.1 the local solution belongs to $\mathcal{M}(t), t \leq T$. For small $\varepsilon_{1}$ and $\beta$ the existence time $T$ is correspondingly large, so we can assume it is a fixed positive number. To prove the last result we needed the Korn inequalities (see Section 4) and imbedding theorems. The constants in those theorems depend on $\Omega_{t}$, the shape of $S_{t}$ and $\int_{0}^{t}\|v\|_{3, \Omega_{\tau}}^{2} d \tau$, so generally they are functions of $t$.

But in view of (5.1) with sufficiently small $\varepsilon_{1}, \beta$ we obtain

$$
\left|\int_{0}^{t} v d \tau\right| \leq c\left(\varepsilon_{1}+\beta\right), \quad t \in[0, T] .
$$

Hence from the relation

$$
x=\xi+\int_{0}^{t} v(x(\xi, \tau), \tau) d \tau, \quad \xi \in S, t \leq T,
$$

for sufficiently small $\varepsilon_{1}, \beta$ and fixed $T$, the shape of $S_{t}, t \leq T$, does not change too much, so the constants from the imbedding theorems can be chosen independent of time. Now we wish to extend the solution to the interval $[T, 2 T]$. Using Lemma 5.3 and (5.8)-(5.12) we can prove the existence of a local solution in $\mathcal{M}(t), T \leq t \leq 2 T$. To prove

$$
\varphi(2 T) \leq \varepsilon_{1}
$$

we need inequality (5.7), where the constants depend on the constants from the imbedding theorems and Korn inequalities for $t \in[T, 2 T]$. Therefore we have to show that the shape of $S_{t}$ and $\int_{0}^{t}\|v\|_{3, \Omega_{\tau}}^{2} d \tau, t \leq 2 T$, do not change more than for $t \leq T$. Assume that there exists a local solution in the interval $[0, k T]$. Then in view of Lemma 5.2 and (5.8)-(5.12) we have, 
for $t \in[0, k T]$,

$$
\begin{aligned}
& \left|\int_{0}^{t} v d \tau\right| \leq \int_{0}^{t}\|v\|_{2, \Omega_{\tau}} d \tau \leq c_{1} \sum_{i=0}^{k-1} \int_{i T}^{(i+1) T}\|v\|_{2, \Omega_{t}} d t \\
& \leq c_{1} T^{1 / 2} \sum_{i=0}^{k-1}\left(\int_{i T}^{(i+1) T}\|v\|_{2, \Omega_{t}}^{2} d t\right)^{1 / 2} \leq c_{1} T^{1 / 2} \sum_{i=0}^{k-1}\left(\int_{i T}^{(i+1) T} \varphi(t) d t\right)^{1 / 2} \\
& \leq c_{1} T^{1 / 2} \sum_{i=0}^{k-1} \sqrt{2}\left[\frac{c}{\mu-1 / 2} e^{-i T \mu}+\varphi(i T)\right]^{1 / 2} \\
& \leq \frac{c T^{1 / 2}}{1-e^{-T / 4}}\left[\frac{1}{(\mu-1 / 2)^{1 / 2}}\left(1+\frac{1}{1-e^{-T / 4}}\right)+\varphi^{1 / 2}(0)\right] \equiv I .
\end{aligned}
$$

From (5.8),

$$
\varphi((i+1) T) \leq \frac{c}{\mu-1 / 2} e^{-(i+1) T / 2}+\varphi(i T) e^{-T / 2}, \quad i=0,1, \ldots, k-1,
$$

we have

$$
\begin{aligned}
& \sum_{i=0}^{k-1} \varphi(i T) \leq \frac{\varphi(0)}{1-e^{-T / 2}}+\frac{c}{\mu-1 / 2} \frac{e^{-T / 2}}{1-e^{-T / 2}}+\frac{c}{\mu-1 / 2} \frac{e^{-T}}{1-e^{-T / 2}}+\cdots \\
& \quad+\frac{c}{\mu-1 / 2} \frac{e^{-n T / 2}}{1-e^{-T / 2}}+\cdots \leq \frac{1}{1-e^{-T / 2}}\left(\varphi(0)+\frac{c e^{-T / 2}}{(\mu-1 / 2)\left(1-e^{-T / 2}\right)}\right) .
\end{aligned}
$$

Therefore,

$$
\begin{aligned}
\int_{0}^{t}\|v\|_{3, \Omega_{\tau}}^{2} d \tau & \leq \sum_{i=0}^{k-1} \int_{i T}^{(i+1) T}\|v\|_{3, \Omega_{\tau}}^{2} d \tau \leq \sum_{i=0}^{k-1} \int_{i T}^{(i+1) T} \phi d t \\
& \leq 2 \sum_{i=0}^{k-1}\left(\frac{c}{\mu} e^{-\mu i T}+\varphi(i T)\right) \\
& \leq \frac{c}{1-e^{-T / 2}}\left(\frac{1}{\mu}+\varphi(0)+\frac{1}{(\mu-1 / 2)\left(1-e^{-T / 2}\right)}\right) .
\end{aligned}
$$

We have to underline the importance of the fact that the quantity $\int_{0}^{t} v d \tau$ is sufficiently small for all $t$. We have $\operatorname{dist}\left\{S_{t}, S_{0}\right\} \leq\left|\int_{0}^{t} v d \tau\right| \leq I$, where $I$ is defined in (5.17), so for small $I$ the domains $\Omega_{t}$ and $\Omega$ are close to each other, hence all the imbedding theorems applied and results for elliptic problems (3.10), (3.12), (3.14) are valid for all $\Omega_{t}, t>0$.

Taking $k=2, \varepsilon_{1}$ sufficiently small and $\mu$ sufficiently large we see that $\int_{0}^{t} v(x(\xi, t), t) d t$ is small for any $t \in[0,2 T]$, so (5.17) and (5.18) imply that the shape of $S_{t}$ and $\int_{0}^{t}\|v\|_{3, \Omega_{\tau}}^{2} d \tau$ change no more than in $[0, T]$, and then the differential inequality (3.23) can also be shown for this interval with the same 
constants. Hence in view of Lemma 5.1 the solution of (1.1)-(1.7) belongs to $\mathcal{M}(t), t \in[T, 2 T]$. Next Lemmas 5.1-5.3 and (5.8)-(5.12) imply (5.17).

Repeating the above considerations for the intervals $[k T,(k+1) T]$, $k \geq 2$, we prove the existence for all $t \in \mathbb{R}_{+}$.

Acknowledgments. The author thanks Prof. W. Zajaczkowski for fruitful discussions during the preparation of this paper.

\section{References}

[1] P. Kacprzyk, Local existence of solutions of the free boundary problem for the equations of magnetohydrodynamic incompressible fluid, Appl. Math. (Warsaw) 30 (2003), 461-488.

[2] - Almost global existence of solutions of the free boundary problem for the equations of magnetohydrodynamic incompressible fluid, ibid. 31 (2004), 69-77.

[3] O. A. Ladyzhenskaya and V. A. Solonnikov, On some problems of vector analysis and generalized formulations of boundary problems for Navier-Stokes equations, Zap. Nauchn. Sem. LOMI 59 (1976), 81-116 (in Russian).

[4] V. A. Solonnikov, On an unsteady motion of an isolated volume of a viscous incompressible fluid, Izv. Akad. Nauk SSSR Ser. Mat. 51 (1987), 1065-1087 (in Russian).

[5] - Solvability of a problem on the motion of a viscous incompressible fluid bounded by a free surface, ibid. 41 (1977), 1368-1424 (in Russian).

[6] - Estimates of solutions of an initial-boundary value problem for the linear nonstationary Navier-Stokes system, Zap. Nauchn. Sem. LOMI 59 (1976), 178-254 (in Russian).

[7] - On the solvability of the second initial-boundary value problem for the linear nonstationary Navier-Stokes system, ibid. 69 (1977), 200-218 (in Russian).

Institute of Mathematics and Cryptology

Cybernetics Faculty

Military University of Technology

S. Kaliskiego 2

00-908 Warszawa, Poland

E-mail: pkacprzyk@wat.edu.pl

Received on 16.11.2005;

revised version on 26.1.2007 
